\title{
Comment:
}

\section{School mental health: Integrating Youth Voices to Shift the Paradigm}

Mina Fazel and Kimberly Hoagwood

Deteriorating patterns of pupil mental health are emerging across the globe, exacerbated by the additional pressures of the Covid-19 pandemic which is affecting access to educational and employment opportunities for young populations. Finding ways to support pupils in their schools given the myriad difficulties they face requires clearly conceptualised public health models built on a solid scientific basis. Failure to implement these models frustrates many, given how the vast majority of children have contact with schools, as opposed to the limited accessibility of mental health services. In addition, many families are desperately trying to seek support for their children, but are unable to access any care, either because it is not available or the pupil is not 'bad enough' to meet mental health service referral criteria. These frustrations by pupils and their families are mirrored by what teachers experience, who often feel they are able to identify pupils who need additional support for mental, emotional, or behavioural problems, but who, despite early identification, cannot harness or access needed supportive services in a timely way.

Although the prevention agenda is a high priority for school mental health, and effective broad-based interventions exist, the science on implementing these interventions so that they are delivered with fidelity and are sustained remains limited. ${ }^{1}$ It is therefore commendable and noteworthy that Cooper et al have been able to conduct a trial trying to fill some of the many gaps in the literature. ${ }^{2}$ They studied a manualised form of individual 
humanistic counselling, demonstrating how to conduct robust research in the school context. This is an important contribution and it helps to move the field of school mental health ahead. The lack of an active control group, however, limits the generalisability of their findings.

The underlying theoretical assumptions for traditional school mental health programs are derived from the same models that have given rise to clinic-based therapies. There is often a reflex that the best intervention for a pupil with early evidence of mental, emotional or behavioural problems is that they need some variation on the theme of a clinic-based intervention, usually an individual talking therapy, be that counselling, solution-focused brief therapy, cognitive behavioural therapy, or interpersonal therapy amongst those offered. This individualistic, medical model, while potentially effective for certain pupils, does not reflect nor take into account the ecology of the school with all of its inherent strengths and opportunities. ${ }^{3}$

As a result, there is a critical public health need to study alternatives to clinic-based models of provision in schools, utilising the range of structured and non-structured educational and interpersonal opportunities that the school environment offers. ${ }^{4,5}$ The responsibility of these ecologically-oriented school mental health approaches, in contrast to more narrowlyfocused clinic-based models, is to encompass families, youth, and the broader community as well, and therefore to armour pupils to develop skills and relationships that enhance their self-esteem and sense of belonging ${ }^{6}$ and that will enable them to manage the transition to adulthood.

One hallmark of the ecologically-oriented public health approaches to school mental health is inclusion of family and/or youth voices. Unfortunately, the absence of the pupil voice is marked in many published studies in school mental health, including the paper by Cooper et 
al. ${ }^{2}$ The need to ensure that developed services are fully informed by stakeholders is foundational to this paradigm. What role did pupils play in developing the intervention? What impressions did they have of its acceptability and efficacy? Such breadth to the research might have helped to explain the higher number of adverse events in the counselling intervention arm in both serious and non-serious events. ${ }^{2}$

We have entered a new stage where young people's participation in the development and assessment of an intervention needs to be at the forefront. ${ }^{7}$ The OxWell School survey in 2019, where 1700 secondary aged pupils completed self-report measures on a range of well-being and mental health questions, asked pupils if they knew how to access mental health support at schools. ${ }^{8}$ Almost three quarters of respondents said they knew how to do so. However, when they were then asked if they would actually seek these services if they needed them (for example, to help them with an emotional difficulty) only $28 \%$ of the respondents said that they would do so. This highlights the dramatic acceptability chasm that exists within the school context and the misalignment of service provision with what young people actually feel they need and would access.

So yes individualized "talking" therapies are useful and needed, but to ensure acceptability, implementation, and sustainability of services, we need to incorporate the perspectives not only of teachers and families, but of pupils themselves, using, for example, participatory and ecologically-based research methodologies. ${ }^{9}$ Inclusion of the perspectives of the endusers-in this case, the youth themselves--improves the science and strengthens the ethics of our scientific work. And we need to broaden our paradigm beyond talking interventions, to integrate public-health models of care, that take full advantage of the naturalistic resources available in school communities, both within and outside the classroom, to ensure 
that youth and family voices are not only heard but also reflected in the services that are offered.

1. Fazel M, Hoagwood K, Stephan S, Ford T. Mental health interventions in schools in high-income countries. Lancet Psychiatry 2014; 1(5): 377-87.

2. Cooper M, Stafford M, Saxon D, et al. Counselling versus Treatment as Usual for Psychological Distress in Adolescents in Schools: Individually Randomised Controlled Trial (ETHOS). Lancet Child Adolesc Health IN PRESS.

3. Atkins MS, Hoagwood KE, Kutash K, Seidman E. Toward the integration of education and mental health in schools. Adm Policy Ment Health 2010; 37(1-2): 40-7.

4. Atkins MS, Lakind D. Usual care for clinicians, unusual care for their clients:

Rearranging priorities for children's mental health services. Adm Policy Ment Health 2013; 40(1): 48-51.

5. Fazel M, Kohrt BA. Prevention versus intervention in school mental health. Lancet Psychiatry 2019; 6(12): 969-71.

6. Weist MD, Hoover S, Lever N, et al. Testing a Package of Evidence-Based Practices in School Mental Health. School Ment Health 2019: 1-15.

7. Sellars E, Pavarini G, Michelson D, Creswell C, Fazel M. Young people's advisory groups in health research: scoping review and mapping of practices. Arch Dis Child 2020. 8. $\quad$ Foster P, Husband D, Dent P, Mansfield KL. Oxfordshire OxWell School Survey 2019: Summary Report. 2019. https://www.psych.ox.ac.uk/files/research/2019-oxwell-schoolsurvey.

9. Shamrova DP, Cummings CE. Participatory action research (PAR) with children and youth: An integrative review of methodology and PAR outcomes for participants, organizations, and communities. Child Youth Serv Rev 2017; 81: 400-12. 